\title{
Sodium ion channel mutations in glioblastoma patients correlate with shorter survival
}

\author{
Avadhut D Joshi ${ }^{1}$, D Williams Parsons ${ }^{2,3}$, Victor E Velculescu ${ }^{2}$, Gregory J Riggins ${ }^{1,2^{*}}$
}

\begin{abstract}
Background: Glioblastoma Multiforme (GBM) is the most common and invasive astrocytic tumor associated with dismal prognosis. Treatment for GBM patients has advanced, but the median survival remains a meager 15 months. In a recent study, 20,000 genes from 21 GBM patients were sequenced that identified frequent mutations in ion channel genes. The goal of this study was to determine whether ion channel mutations have a role in disease progression and whether molecular targeting of ion channels is a promising therapeutic strategy for GBM patients. Therefore, we compared GBM patient survival on the basis of presence or absence of mutations in calcium, potassium and sodium ion transport genes. Cardiac glycosides, known sodium channel inhibitors, were then tested for their ability to inhibit GBM cell proliferation.
\end{abstract}

Results: Nearly $90 \%$ of patients showed at least one mutation in ion transport genes. GBM patients with mutations in sodium channels showed a significantly shorter survival compared to patients with no sodium channel mutations, whereas a similar comparison based on mutational status of calcium or potassium ion channel mutations showed no survival differences. Experimentally, targeting GBM cells with cardiac glycosides such as digoxin and ouabain demonstrated preferential cytotoxicity against U-87 and D54 GBM cells compared to nontumor astrocytes (NTAs).

Conclusions: These pilot studies of GBM patients with sodium channel mutations indicate an association with a more aggressive disease and significantly shorter survival. Moreover, inhibition of GBM cells by ion channel inhibitors such as cardiac glycosides suggest a therapeutic strategy with relatively safe drugs for targeting GBM ion channel mutations. Key Words: glioblastoma multiforme, ion channels, mutations, small molecule inhibitors, cardiac glycosides.

\section{Background}

Glioblastoma multiforme (GBM) is a malignant astrocytic brain tumor with a current median survival of about 15 months [1]. The current standard of care therapy is surgery followed by concurrent radiation plus temozolomide. The addition of the DNA alkylating agent temozolomide improves the survival by 10 weeks. There is a similar increase in survival with local delivery of BCNU, the other currently approved chemotherapy for this tumor [2]. The survival gains for GBM patients show that progress can be made, but this progress has been slow. It is not clear if large survival gains can be

\footnotetext{
* Correspondence: griggin1@jhmi.edu

'Department of Neurosurgery, Johns Hopkins University Medical School, Baltimore, MD, 21231, USA

Full list of author information is available at the end of the article
}

achieved with the current trio of radiation, surgery and DNA damaging chemotherapy.

Recently, GBMs have undergone a large-scale mutation screen [3] and the molecular targets for this cancer can be re-evaluated. Critical to this approach is the identification of altered proteins or pathways that initiate and/or promote tumor growth. Ideally, these molecular targets are unique to the tumor cell, and therapy specific to the alteration does not harm normal cells. There are some very well known genes mutated in GBM such as the tumor suppressors $p 53$ and PTEN, and amplification or mutation of the EGFR and PDGFRA oncogenes. Unfortunately, molecular targeting efforts in GBM so far have not been translated into clinical success, despite some promising results of targeted therapy in a few other cancers.

\section{Biomed Central}

(C) 2011 Joshi et al; licensee BioMed Central Ltd. This is an Open Access article distributed under the terms of the Creative Commons Attribution License (http://creativecommons.org/licenses/by/2.0), which permits unrestricted use, distribution, and reproduction in any medium, provided the original work is properly cited. 
Although there are many possible reasons why molecular targeting has not yet been successful in GBM, it is possible that different or additional molecular targets in combination will have better success. A recent survey of the coding sequence of 20,661 genes in GBM genomes has implicated many new mutated genes [3]. Similar to other cancers there are many mutated genes in GBM and these genes cluster into key pathways or gene groups. This clustering occurs more than chance predicts, suggesting that these are a small number of key cellular processes that need to be altered in the majority GBMs. One cluster of mutated genes reported by Parsons et al. [3] was the ion channel genes. Of the 555 genes involved in sodium, potassium, calcium and other ion transport, 55 mutations were detected affecting 90\% of the samples studied with at least one somatic mutation. The statistical significance of this observation was estimated to be $\mathrm{p}<0.001$ and the ion channels were ranked as one of the top gene clusters implicated by acquired mutations in GBM.

Ion channels form a crucial part of cellular machinery and are responsible for transporting essential ions across cell membranes, maintaining cell shape, cell volume and plasma membrane potential [4-6]. Recent evidence suggests a role for ion channels in cancer progression and metastasis [7-9]. Ion channels, such as sodium channels, potassium channels and calcium channels, have been implicated for their role in a number of different cancers such as colon cancer, prostate cancer, breast cancer and lung cancer $[8,9]$. For example, the up-regulation of voltage gated sodium channels is associated with progression of breast cancer metastasis [10].

In this study, we report a correlation between ion channel mutations and patient survival. Twenty-one GBM patients where sodium, potassium and calcium channel gene sequences were known [3] were analyzed further for this study. GBM patients with a mutation in any of the sodium channel genes had a significantly shorter survival compared to those with wild-type sequence. In contrast, there was no statistical survival difference for GBM patients with either potassium or calcium channel mutations. We extended these findings with a preliminary in vitro laboratory investigation to determine if known sodium channel inhibitors had an effect on GBM cells relative to cells with a normal genome.

\section{Patients and Methods}

\section{Patient Characteristics}

The twenty-one patients included in this study were those analyzed for mutations in 20,661 genes in a previous study [3]. There were eight females and 13 males. Median age of GBM patients was 45 years (median age in males 45 years, median age in females 60 years; not significantly different). None of the GBM patients had received a prior chemo or a radiation therapy. One of the 21 patients was diagnosed as a ganglioglioma patient but was associated with a later recurrence of GBM. Median survival of the patients was 54.9 weeks (range 2 - 215 weeks). The characteristics of patient cohort are summarized in Table 1.

\section{GBM Patient Samples and Genome Sequencing}

The GBM sequencing results have been previously published by Parsons et al. [3]. GBM patient tumor samples were obtained using an IRB approved protocols. Twenty-one GBM samples consisting of six surgically resected patient tumors and 15 samples were passaged in nude mice as xenografts. These 21 samples were amplified by PCR for sequence analysis. Primer pairs were used to amplify and sequence 175,471 coding exons and adjacent intronic splice donor and acceptor sequences in $21 \mathrm{GBM}$ samples and one matched normal sample as described previously [3].

\section{Gene Selection}

All the mutated genes were classified according to gene ontology into different gene sets [3]. Ion channel classification used in this study included voltage gated ion channels and ion co-transporters and are referred to as channels for sake of simplicity. All the genes involved in ion transport including, sodium channels, potassium channels, calcium channels were selected and genes specifically associated with sodium channels, potassium channels and calcium channels were selected individually from these sets.

\section{Cell Culture}

U-87 GBM cells were obtained from ATCC and D54 GBM cells were obtained from Duke University Medical Center. Both U87 and D54 cells were maintained in DMEM supplemented with $10 \%$ FBS and penicillin/ streptomycin. Non-tumor astrocytes (NTAs) consisted of brain cortex tissue surgically resected from epilepsy patients using an IRB approved protocol. The cortex was cut into fine pieces using a scalpel and was cultured for less than 8 passages in DMEM F12 cell culture medium supplemented with $10 \%$ FBS and penicillin/ streptomycin.

\section{Cell Proliferation and Drug Sensitivity Assay}

The cardiac glycosides digoxin and ouabain (SigmaAldrich) were dissolved in methanol and phosphate buffered saline (PBS), respectively, to make a stock solution of $25 \mathrm{mM}$. Subsequent dilutions were made from this stock solution. Methanol and PBS were used as a vehicle control for digoxin and ouabain respectively, during the proliferation assay. Cell proliferation was assessed using 
Table 1 GBM patient characteristics

\begin{tabular}{|c|c|c|c|c|c|c|c|c|c|c|c|c|}
\hline $\begin{array}{l}\text { Tumor } \\
\text { ID }\end{array}$ & $\begin{array}{c}\text { Patient } \\
\text { age } \\
\text { (years) }\end{array}$ & Sex & Pathology & $\begin{array}{l}\text { Recurrent } \\
\text { GBM }\end{array}$ & $\begin{array}{l}\text { Secondary } \\
\text { GBM }\end{array}$ & $\begin{array}{c}\text { Prior } \\
\text { radiation } \\
\text { therapy }\end{array}$ & $\begin{array}{c}\text { Prior } \\
\text { chemotherapy }\end{array}$ & $\begin{array}{l}\text { Survival after tumor } \\
\text { sample obtained (Days) }\end{array}$ & $\begin{array}{l}\text { Sample } \\
\text { type }\end{array}$ & $\begin{array}{l}\text { Sodium } \\
\text { channel } \\
\text { mutations }\end{array}$ & $\begin{array}{l}\text { Potassium } \\
\text { channel } \\
\text { mutations }\end{array}$ & $\begin{array}{c}\text { Calcium } \\
\text { channel } \\
\text { mutations }\end{array}$ \\
\hline $\mathrm{BrO2X}$ & 39 & $M$ & GBM & No & No & No & No & Unknown & $X G$ & Yes & No & Yes \\
\hline $\mathrm{Br03X}$ & 44 & $M$ & GBM & No & No & No & No & 422 & $X G$ & Yes & Yes & No \\
\hline Br04X & 45 & $F$ & GBM & Yes & No & NA & NA & Unknown & $X G$ & No & No & No \\
\hline Br05X & 41 & $M$ & GBM & No & No & No & No & 563 & $X G$ & No & No & Yes \\
\hline Br06X & 11 & $M$ & GBM & No & No & No & No & 986 & $X G$ & No & No & No \\
\hline Br07X & 45 & $M$ & GBM & No & No & No & No & 350 & $X G$ & Yes & No & Yes \\
\hline Br08X & 54 & $M$ & HGG & No & No & No & No & 384 & $X G$ & No & Yes & No \\
\hline Br09P & 51 & $M$ & GBM & No & No & No & No & 588 & PT & Yes & Yes & Yes \\
\hline Br10P* & 30 & $F$ & GBM & No & No & No & No & 813 & PT & No & No & Yes \\
\hline $\mathrm{Br11P^{* }}$ & 32 & $M$ & GBM & No & No & No & No & 1502 & PT & No & Yes & No \\
\hline $\mathrm{Br} 12 \mathrm{P}^{*}$ & 31 & $M$ & GBM & No & No & No & No & 566 & PT & No & No & Yes \\
\hline$B r 13 X$ & 59 & $F$ & GBM & No & No & No & No & 174 & $X G$ & Yes & Yes & Yes \\
\hline $\operatorname{Br} 14 X$ & 61 & $F$ & GBM & No & No & No & No & Unknown & $X G$ & No & Yes & Yes \\
\hline $\operatorname{Br} 15 X$ & 61 & $M$ & GBM & No & No & No & No & 56 & $X G$ & Yes & No & Yes \\
\hline Br16X & 63 & $M$ & GBM & No & No & No & No & Unknown & $X G$ & Yes & No & Yes \\
\hline Br17X & 63 & $M$ & GBM & No & No & No & No & 964 & $X G$ & No & Yes & Yes \\
\hline $\mathrm{Br} 20 \mathrm{P}$ & 77 & $F$ & GBM & No & No & No & No & 122 & PT & Yes & Yes & Yes \\
\hline Br23X & 78 & $F$ & GBM & No & No & No & No & 16 & $X G$ & Yes & No & No \\
\hline $\operatorname{Br} 25 X$ & 45 & $M$ & GBM & No & No & No & No & 48 & $X G$ & Yes & No & No \\
\hline Br26X & 66 & $F$ & GBM & No & No & No & No & 61 & $X G$ & No & Yes & No \\
\hline Br29P & 42 & $\mathrm{~F}$ & HGG & Yes & NA & NA & NA & Unknown & PT & Yes & Yes & No \\
\hline
\end{tabular}

*Patients with IDH1 mutations. 
an alamarBlue ${ }^{\circledR}$ assay (Invitrogen, Carlsbad, CA). U-87, D54 and NTAs were plated (1000 cells/well) in black clear bottom 96 well plates (Becton Dickinson, Bedford MA) and incubated overnight. The following day, each drug was added at its designed concentration with $20 \mu \mathrm{l}$ of 10X alamarBlue reagents. The volume in each well was made up to $200 \mu \mathrm{l}$ with the growth medium. After 72 hours incubation, alamarBlue fluorescence was measured on a Perkin Elmer Wallac 1420 Multilabel counter (Perkin Elmer, Turku, Finland) with a $540 \mathrm{~nm}$ excitation filter and a $590 \mathrm{~nm}$ emission filter. Fold inhibition was calculated by dividing the fluorescence values for control cells (cells treated with vehicle) with fluorescence values of cells treated with a particular concentration of cardiac glycosides. For apoptosis analysis, both U-87 and NTAs were plated in a six well plate $(100,000$ cells/well $)$ and incubated for 24 hours. After 24 hours, cells were treated with $500 \mathrm{nM}$ of digoxin and ouabain overnight and observed under a microscope

\section{Statistical Analysis}

GraphPad Prism 5 software was used to compute all the survival curves. To determine the clinical outcome, patient survival was used as a measure where survival was defined as the time in days from first surgical resection of GBM to death. Out of 21 patient samples, survival data were available for 16 different patients.

\section{Results}

\section{Mutations in Sodium Ion Channels are Associated with} Shorter Survival in GBM Patients

Systematic analyses of functional gene groups and pathways from a previous study [3] identified ion channel genes that transport sodium, potassium or calcium ions as one of the gene groups most frequently mutated in GBM. The sodium, potassium and calcium ion transport gene groups were each evaluated to determine if mutations in these gene groups altered average patient survival. Nineteen of the 21 patients (90\%) showed at least one mutation in sodium, potassium or calcium channels taken together. Fourteen sodium channel genes (Table 2), 13 potassium channel genes (Table 3 ) and 18 calcium channel genes (Table 4) had somatic mutations. None of the mutations were found in more than one patient except for SCN9A, CACNA1H, and TRPV5 where each gene was mutated in two patients. Interestingly, all the samples with IDH1 mutations did not have any sodium channel mutations. A comprehensive list of genes was divided into individual lists of genes that were associated with sodium channels, potassium channels or calcium channels (Tables 2, 3 and 4). For example, patients were classified into the sodium channel mutation group if they had a mutation in at least one sodium channel gene (listed in Table 2). If there were no mutations in any sodium channels, the patients were grouped into a sodium channel 'unmutated' group. Patients were grouped in a similar way for potassium channels and calcium channels.

To determine the role of individual ion channels in GBM patients, survival of GBM patients was compared using Kaplan Meier analyses. GBM patients with sodium channel mutations showed a significantly shorter survival $(\mathrm{p}=0.0079)$ compared to patients with unmutated sodium channels. Median survival of GBM patients with mutated sodium channels was 148 days compared to 689 days in patients with no sodium channel mutations (Figure 1A). A similar comparison in GBM patients with

Table 2 Sodium channel mutations

\begin{tabular}{|c|c|c|c|c|c|c|}
\hline Gene & Transcript Accession & Tumor & Nucleotide (genomic) & Nucleotide (cDNA) & Amino acid (protein) & Mutation Type \\
\hline ATP12A & NM_001676 & $\operatorname{Br} 13 X$ & g.chr13:24178566C>G & C. $2134 C>G$ & p.Q712E & Missense \\
\hline SCN1B & CCDS12441.1 & $\mathrm{BRO} 2 \mathrm{X}$ & g.chr19:40213563C>T & UTR-2C>T & 5'UTR & $5^{\prime} \cup T R$ \\
\hline SCN3A & NM_006922 & Br03x & g.chr2:165772558G >A & c. $5612 \mathrm{G}>\mathrm{A}$ & p.R1871 Q & Missense \\
\hline SCN3B & CCDS8442. 1 & Br16X & g.chr11:123018465_12301 8464delCT & c.344_345del CT & fs & INDEL \\
\hline SCN5A & NM_000335 & Br20P & g.chr3:38579032G>T & c.3838G $>\mathrm{T}$ & p.V1280 F & Missense \\
\hline SCN9A & NM_002977 & Br9PT & g.chr2:166987822G $>A$ & c. $583 G>A$ & p.V195I & Missense \\
\hline SCN9A & NM_002977 & Br07X & g.chr2:166881761C>T & c. $4862 C>T$ & p.T1621 M & Missense \\
\hline SLC11A1 & CCDS2415. 1 & $\operatorname{Br} 25 X$ & g.chr2:219077407G >A & C. $520 \mathrm{G}>\mathrm{A}$ & p.V174l & Missense \\
\hline SLC1A2 & NM_004171 & BrO3X & g.chr11:35239082_352390 81delCT & c.1660_1661 delCT & fs & INDEL \\
\hline SLC5A7 & CCDS2074. 1 & Br15X & g.chr2:108067164C>A & C. $263 C>A$ & p.P88Q & Missense \\
\hline SLC8A1 & CCDS1806. 1 & Br07X & g.chr2:40568624C>G & c. $448 C>G$ & p.L150V & Missense \\
\hline SLC9A1 & CCDS295.1 & Br07X & g.chr1:27120218C>T & c. $1006 C>T$ & p.L336F & Missense \\
\hline SLC9A2 & CCDS2062. 1 & Br23X & g.chr2:102732730G $>A$ & c. $479 \mathrm{G}>\mathrm{A}$ & p.R160H & Missense \\
\hline SLC9A4 & NM_001011552 & Br9PT & g.chr2:102583058G >A & c.1201G $>A$ & p.V401I & Missense \\
\hline TRPM5 & NM_014555 & Br29P & g.chr11:2382782G>A & c.3459G $>A$ & p.W1153X & Missense \\
\hline
\end{tabular}


Table 3 Potassium channel mutations

\begin{tabular}{|c|c|c|c|c|c|c|}
\hline Gene & Transcript Accession & Tumor & Nucleotide (genomic)* & Nucleotide (cDNA) & Amino acid (protein) & Mutation Type \\
\hline ATP12A & NM_001676 & $\operatorname{Br13X}$ & g.chr13:24178566C>G & c. $2134 C>G$ & p.Q712E & Missense \\
\hline GRIK4 & CCDS8433.1 & $\operatorname{Br17X}$ & g.chr11:120343207G>A & c. $2360 G>A$ & p.G787E & Missense \\
\hline KCNA4 & NM_002233 & Br29P & g.chr11:29990620C>T & c. $182 C>T$ & p.S61F & Missense \\
\hline KCNB2 & CCDS6209.1 & $\mathrm{Br} 11 \mathrm{P}$ & g.chr8:74011087G>A & c. $943 G>A$ & p.A315T & Missense \\
\hline KCND2 & CCDS5776.1 & Br03X & g.chr7:119979914C>T & C.1597C >T & p.R533X & Missense \\
\hline KCNG3 & CCDS1809.1 & Br26X & g.chr2:42631881G>A & c. $412 \mathrm{G}>\mathrm{A}$ & p.D138N & Missense \\
\hline $\mathrm{KCNH1}$ & CCDS1496.1 & Br9PT & g.chr1:207365704G>T & c. $1662 \mathrm{G}>\mathrm{T}$ & p.K554N & Missense \\
\hline $\mathrm{KCNH5}$ & CCDS9756.1 & Br08X & g.chr14:62316201C>T & c. $2017 C>T$ & p.R673W & Missense \\
\hline KCNJ15 & CCDS13656.1 & $\mathrm{BrO3X}$ & g.chr21:38593138G>A & $c .85 G>A$ & p.V29l & Missense \\
\hline KCNK1 & CCDS1599.1 & Br26X & g.chr1:230109198G>A & c. $478 \mathrm{G}>\mathrm{A}$ & p.V160l & Missense \\
\hline LRRC4B & ENST00000253728 & Br03X & g.chr19:55713328C>T & C.809C $>\mathrm{T}$ & p.T270l & Missense \\
\hline REN & NM_000537 & $\mathrm{Br} 20 \mathrm{P}$ & g.chr1:200862821G>A & c. $226 \mathrm{G}>\mathrm{A}$ & p.V76M & Missense \\
\hline SLC12A5 & CCDS13391.1 & $\operatorname{Br14X}$ & g.chr20:44097883G>A & c.340G $>A$ & p.V114| & Missense \\
\hline
\end{tabular}

mutated and unmutated potassium channels (Figure 1B), and mutated and unmutated calcium channels (Figure 1C) showed no significant difference in survival. These observations suggest that survival difference seen in GBM patients with mutated and unmutated sodium channels are not random.

GBM patients with PTEN mutations are associated with shorter survival [11]. Therefore, to rule out the effect of PTEN mutation on the survival curves with mutated and unmutated sodium channels, we excluded the patients with PTEN mutations. In spite of the exclusion of patients with PTEN mutation, GBM patients with sodium channel mutations were associated with significantly shorter survival (Figure 1D). Median survival of patients with sodium channel mutations was 122 days compared to 566 days in patients with no mutations.

Table 4 Calcium channel mutations

\begin{tabular}{|c|c|c|c|c|c|c|}
\hline Gene & Transcript Accession & Tumor & Nucleotide (genomic)* & Nucleotide (cDNA) & Amino acid (protein) & Mutation Type \\
\hline ATP2B1 & CCDS9035.1 & $\mathrm{Br} 12 \mathrm{P}$ & g.chr12:88531401T>A & C. $502 T>A$ & p.L168I & Missense \\
\hline CACNA1A & NM_000068 & $\mathrm{Br} 15 \mathrm{X}$ & g.chr19:13184518C>T & C. $5980 C>T$ & p.P1994S & Missense \\
\hline CACNA1C & NM_000719 & $\mathrm{Br} 17 \mathrm{X}$ & g.chr12:2094747C>A & c. $146 C>A$ & p.A49D & Missense \\
\hline CACNA1H & NM_021098 & $\mathrm{Br} 15 \mathrm{X}$ & g.chr16:1199144C>T & c. $3475 C>T$ & p.Q1159X & Missense \\
\hline CACNA1H & NM_021098 & Br05X & g.chr16:1201735G>A & c. $4495 \mathrm{G}>\mathrm{A}$ & p.V1499M & Missense \\
\hline CACNA2D3 & NM_018398 & Br07X & g.chr3:54905889G $>A$ & c. $2320 G>A$ & p.A774T & Missense \\
\hline CHRNA3 & CCDS10305.1 & $\mathrm{Br} 14 \mathrm{X}$ & g.chr15:76681620T>C & c.419T>C & p.L140S & Missense \\
\hline CHRNA9 & CCDS3459.1 & $\operatorname{Br} 15 X$ & g.chr4:40197220C>T & C.1195C>T & p.R399C & Missense \\
\hline GRIN2B & CCDS8662.1 & $\mathrm{Br} 13 \mathrm{X}$ & g.chr12:13608123G>A & c.3316G $>A$ & p.E1106K & Missense \\
\hline GRM1 & CCDS5209.1 & $\operatorname{Br} 15 X$ & g.chr6:146761918C>T & c. $2050 C>T$ & p.R684C & Missense \\
\hline ITPR3 & CCDS4783.1 & $\mathrm{Br} 16 \mathrm{X}$ & g.chr6:33761264G>A & c.5458G $>A$ & p.E1820K & Missense \\
\hline KIAA0703 & NM_014861 & $\mathrm{Br} 20 \mathrm{P}$ & g.chr16:83006699G $>A$ & IVS5+1G>A & Splice Site & Splice Site \\
\hline NMUR1 & CCDS2486.1 & $\mathrm{Br} 20 \mathrm{P}$ & g.chr2:232219206C >T & $\mathrm{c} .31 \mathrm{C}>\mathrm{T}$ & p.L11F & Missense \\
\hline PKD1L2 & NM_182740 & $\operatorname{Br} 14 X$ & g.chr16:79765857G>T & c. $2747 \mathrm{G}>\mathrm{T}$ & p.G916V & Missense \\
\hline RYR2 & NM_001035 & $\mathrm{Br} 10 \mathrm{P}$ & g.chr1:233820306G>A & c. $256 \mathrm{G}>\mathrm{A}$ & p.V86M & Missense \\
\hline RYR2 & NM_001035 & $\mathrm{Br} 17 \mathrm{X}$ & g.chr1:233858930G $>$ A & c. $365 \mathrm{G}>\mathrm{A}$ & p.R122H & Missense \\
\hline RYR3 & NM_001036 & Br9PT & g.chr15:31893017C>T & c. $10447 C>T$ & p.R3483W & Missense \\
\hline SLC8A1 & CCDS1806.1 & Br07X & g.chr2:40568624C>G & c. $448 C>G$ & p.L150V & Missense \\
\hline STIM2 & CCDS3440.1 & $\mathrm{BrO2X}$ & g.chr4:26686687G $>T$ & c. $1544 \mathrm{G}>\mathrm{T}$ & p.R515L & Missense \\
\hline TRPV5 & CCDS5875.1 & $\mathrm{Br} 15 \mathrm{X}$ & g.chr7:142139519G >A & C.1064G $>A$ & p.R355H & Missense \\
\hline TRPV5 & CCDS5875.1 & Br14X & g.chr7:142139507G>A & c.1076G $>A$ & p.R359H & Missense \\
\hline
\end{tabular}




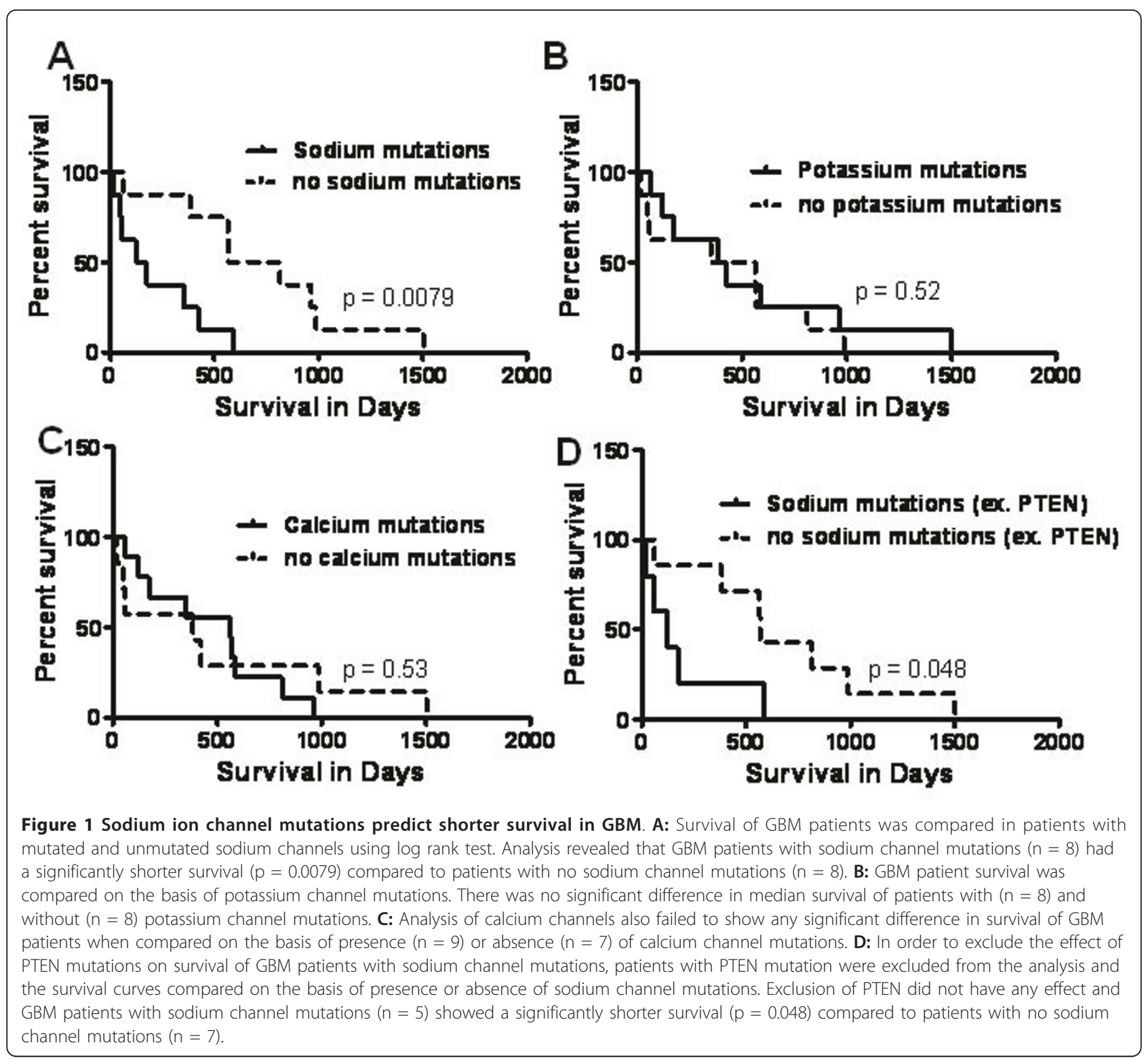

Targeting Ion Channels Preferentially Inhibits Growth of Glioblastoma Cells

Because sodium channel mutations had a substantial effect on GBM patient survival, targeting sodium channels may be an effective way to counter GBM cell growth. We started our study with sodium channel inhibitors with previous clinical use. Based on information in the literature, and a larger screen of libraries of approved drugs (data not shown), we selected two cardiac glycosides, digoxin (FDA approved) and ouabain, to test on GBM cells. Our reasoning for choosing cardiac glycosides was based on two main previously reported findings. First, the anti-proliferative or anticancer effect of cardiac glycosides is well documented [12-14] and second; cardiac glycosides may be neuroprotective [15] and thus, might be used safely in the central nervous system.

The effect of ouabain and digoxin on proliferation of U-87 and D54 GBM cells and NTAs was tested first, using an alamarBlue based assay. Cells were treated at different concentrations ranging from $10 \mathrm{nM}$ to $50 \mu \mathrm{M}$ in a 96 well plate. After 72 hours, both digoxin and ouabain showed preferential anti-proliferation and toxicity against U-87 and D54 GBM cells compared to the NTAs (Figure 2A and 2B). In addition, comparison of growth curves of U-87 and NTAs treated with $500 \mathrm{nM}$ digoxin and ouabain demonstrated a preferential inhibition of U-87 GBM cells over NTAs (Figure 2C and 2D). 


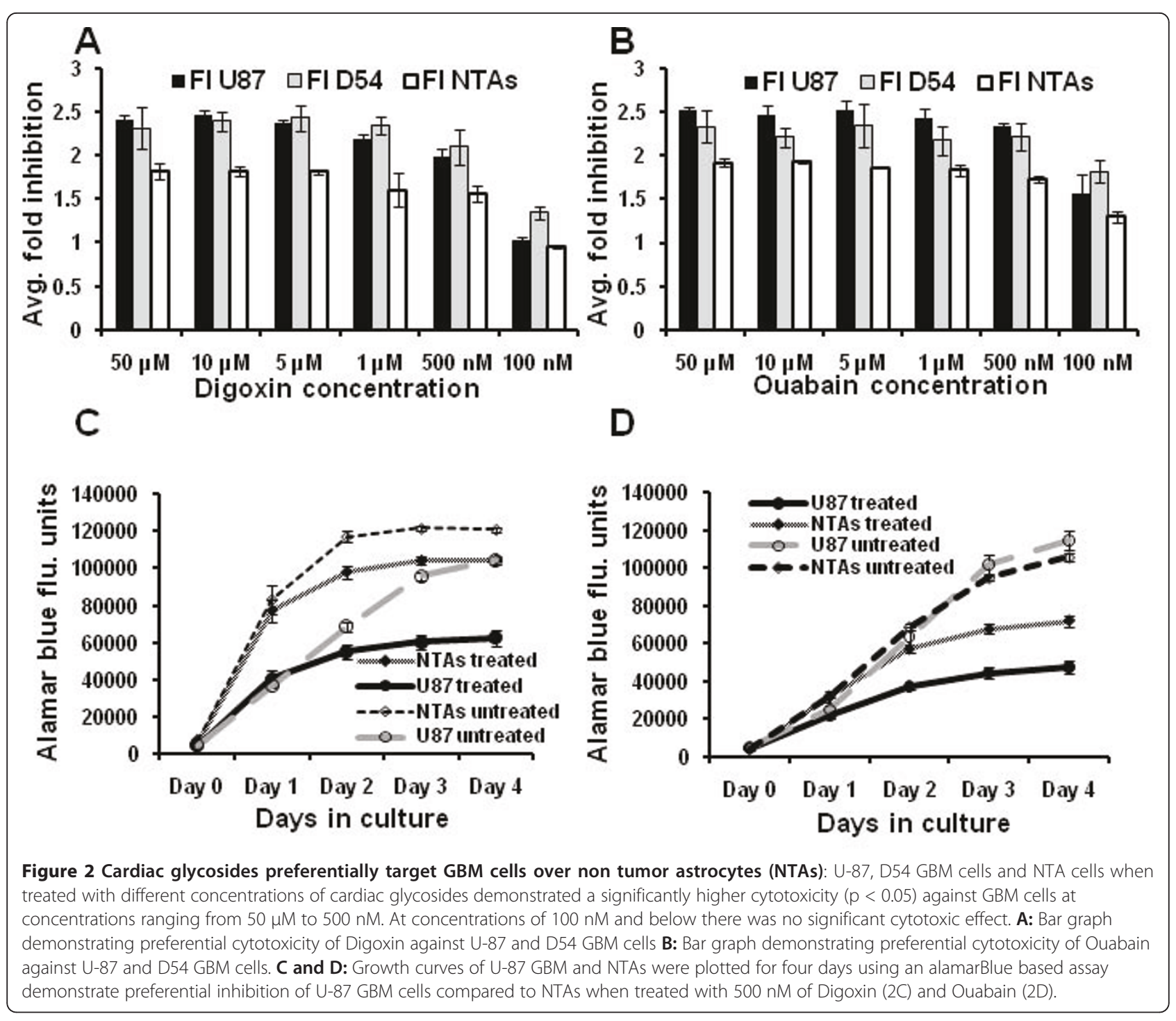

Furthermore, to confirm that GBM cells were preferentially targeted by an alternative technique, U-87 cells and NTAs treated with $500 \mathrm{nM}$ of digoxin and ouabain overnight and were observed under a light microscope next morning. Figure 3 demonstrates that U-87 GBM cells treated with digoxin and ouabain detach and showed an apoptotic phenotype, whereas NTAs remained adherent and did not show an apoptotic phenotype, confirming the preferential cytotoxicity of cardiac glycosides.

\section{Discussion}

In the GBM patients studied those with mutations in the sodium ion channel genes had a significantly shorter survival compared to patients without a mutation. In comparison, similar analyses of mutations in potassium channels and calcium channels showed no statistical survival differences. One biological possible explanation for this observation is that sodium channel mutations promote GBM tumor growth and/or invasion, thereby decreasing survival, whereas other non-sodium ion channel mutations do not function to alter invasion. This is the first report suggesting a possible role of ion channel mutations in GBM prognosis. Nineteen out of 21 (90\%) patient samples showed at least one mutation in sodium, potassium or calcium channels. It will be important to see if this observation can be reproduced in larger studies and/or other patient populations. Furthermore, it was found that patients with no sodium channel mutations were younger (median age 43 years) compared to patients with mutated sodium channel mutations (median age 51 years), although the difference did not turn out to be statistically significant ( $\mathrm{p}=0.117$ ).

Recently, IDH1 mutations have been identified to be associated with a specific subgroup of GBM patients 

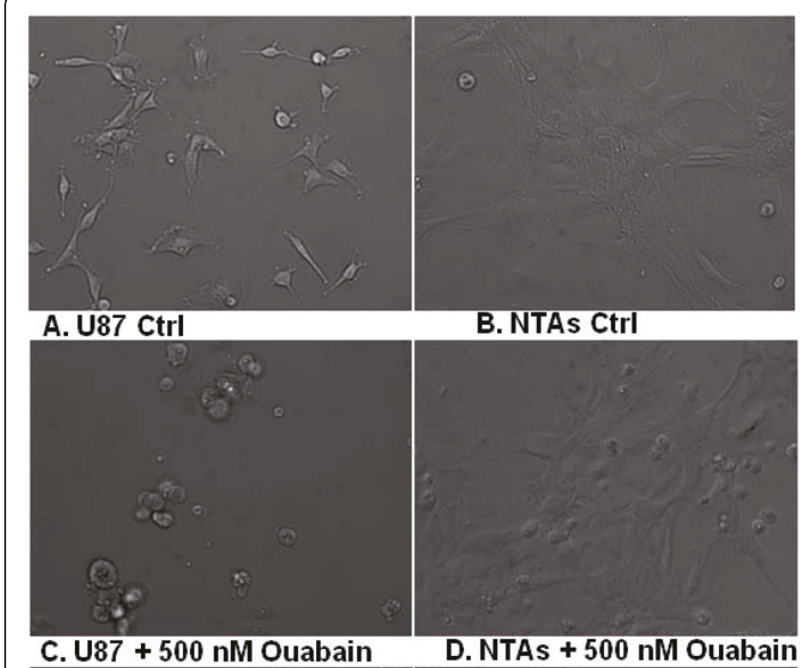

B. NTAs Ctrl

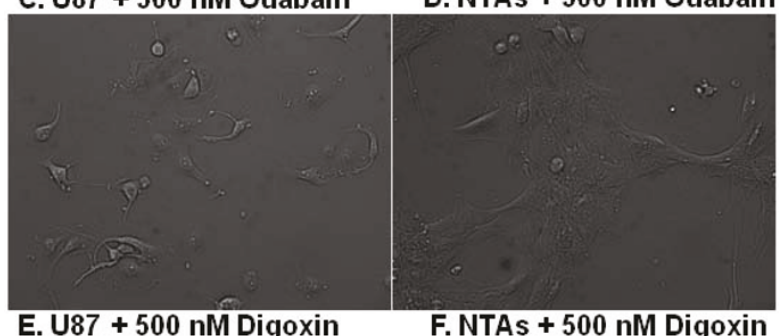

+ 500 nM Digoxin

F. NTAs + 500 nM Digoxin

Figure 3 Light microscope pictures depicting apoptotic phenotype/dead U-87 GBM cells after an overnight treatment with ouabain and digoxin. Non-tumor astrocytes remain

unaffected for most part, although they demonstrated delayed (after 36 hrs) apoptotic phenotype.

who are younger and have a better prognosis $[3,16]$. Interestingly, we found that all the patients with IDH1 mutations were a part of sodium channel unmutated group. However it is not known whether this association is significant because of the small sample size. It also raises the question whether IDH1 mutations would contribute to improved survival in patients with unmutated sodium channels. Analysis of survival data after excluding IDH1 mutated patients revealed that median survival in patients with sodium channel mutations was 148 days compared to 563 days in sodium channel unmutated patients in accordance with our earlier observations, however the $\mathrm{p}$ value dropped to 0.06 (data not shown). These observations warrant a larger and more in depth study to investigate whether there is an association between IDH1 mutation and GBM patients with unmutated sodium channels and whether the improved survival seen in GBM patients with unmutated sodium channels is independent of IDH1 mutation status.

Ion channel genes were mutated at a higher frequency compared to other genes $(\mathrm{p}<0.001)$. Moreover, individual groups of genes consisting of calcium ion transport $(\mathrm{p}<$ $0.001)$, sodium ion transport ( $\mathrm{p}<0.005)$ and potassium ion transport $(\mathrm{p}=0.037)$ showed a significantly higher frequency of mutation [3]. Most of the ion channel genes were mutated only once except for SCN9A, CACNA1H and TRPV5 which were each mutated twice in the set of 21 patients. Since no gene was mutated more than twice in this set and there are many possible ion channels with mutations, this indicates a low mutation rate for each individual gene, despite the group being highly mutated. Subset classes of the ion channels, such as SCN or SLC (both sodium channels) or $\mathrm{KCN}$ (potassium channels), were also enriched for mutations [3]. These observations suggests that mutations in a gene family or molecular pathway of similar function when considered in combination may be more informative than a single gene, when evaluating tumor growth and selecting molecular targets.

In this report, we have used the mutation status of sodium channels as a variable for comparing patient survival. However, which mutations are relevant to GBM biology and how they alter the clinical course of GBM remains unknown. One interpretation of our data is that sodium channel inhibition slows tumor cell growth, suggesting that sodium channel mutations are activating, or activate some mechanism responsible for poor prognosis. However, there is no evidence at the molecular level as to how these mutations might work. Further in depth molecular physiological studies to determine the direct effect of the mutations on membrane potential and polarization/depolarization and cell signaling of the tumor cells would be an option to study this question.

Sodium, potassium and calcium channels form an intricate network that maintains ionic balance in the cell and mutation in any one of the ion channels could alter many cellular functions. One hypothesis is that ion channel mutations are partially responsible for the increased motility of GBM cells. Voltage gated sodium channels have already been implicated for their role in enhancing the invasiveness of breast cancer and prostate cancer [10,17-19]. Higher expression of SCN5A has been associated with higher metastatic potential. It has also been reported that EGF may increase metastatic potential of prostate cancer by up regulation of SCN9A [19]. Although, our data does not describe expression levels of sodium channels, there is a possibility that the mutations in SCN5A and SCN9A may cause an increase in the activity of sodium channels thereby increasing the metastatic potential of GBM and decreasing survival of the patients.

Ion channels might be investigated as a pharmacological target for GBM patient therapy. Our data demonstrates that ion channel inhibitors, cardiac glycosides in this case can preferentially inhibits GBM cells over nontumor astrocytes (NTAs) when tested in vitro. There is no evidence yet that cardiac glycosides molecularly 
interact directly with any of the mutated sodium channel (shown in Table 2). Nevertheless, preferential targeting of GBM cells by cardiac glycosides suggests that ion channels can be targeted and should be evaluated as a therapeutic drug target for treating GBM in the future. Attempts have been made to treat GBMs with cardiac glycosides with modified structures that reduce their cardio-toxicity and increase anti-proliferative capability [20]. Similarly, targeting of $\alpha 1$ subunit of the sodium pump using the siRNA inhibited growth and migration of lung cancer cells [21]. Voltage gated sodium channels have also been targeted in prostate cancer cells with encouraging results [22].

\section{Conclusion}

In summary, we have shown that mutations in sodium channels are associated with an aggressive form of GBM. We also show in vitro growth inhibition by ion channel inhibitors, suggesting that GBM might be targeted using ion channel inhibitors. These observations from different lines of investigation hint that sodium ion channels should be investigated further as a molecular therapeutic target in GBM.

\section{Acknowledgements}

AJ is a recipient of Matt Trainham ABTA postdoctoral fellowship and GJR is the Irving J. Sherman Neurosurgery Research Professor. The research work was funded by R01 NS052507 and the Virginia and D.K. Ludwig Fund for Cancer Research.

\section{Author details}

'Department of Neurosurgery, Johns Hopkins University Medical School, Baltimore, MD, 21231, USA. ${ }^{2}$ Ludwig Center for Cancer Genetics and Therapeutics, and The Howard Hughes Medical Institute at Johns Hopkins Kimmel Cancer Center, Baltimore, MD 21231, USA. ${ }^{3}$ Department of Pediatrics, Section of Hematology-Oncology, Baylor College of Medicine, Houston TX 77030, USA.

\section{Authors' contributions}

AJ analyzed the mutation data on the basis of ion channel mutations, made the Kaplan Meier survival curves, performed the cytotoxicity assays using digoxin and ouabain and wrote the manuscript. DWP generated all the mutation data for the GBM genome project. W conceived the genome sequencing study and critiqued the manuscript. GJR conceived and help design the study, wrote the manuscript and critically revised the manuscript. All authors have read and agreed to submit the manuscript.

\section{Competing interests}

The authors declare that they have no competing interests.

Received: 21 June 2010 Accepted: 11 February 2011

Published: 11 February 2011

\section{References}

1. Stupp R, Hegi ME, van den Bent MJ, Mason WP, Weller M, Mirimanoff RO, Cairncross JG: Changing paradigms-an update on the multidisciplinary management of malignant glioma. Oncologist 2006, 11:165-180.

2. Lawson HC, Sampath P, Bohan E, Park MC, Hussain N, Olivi A, Weingart J, Kleinberg L, Brem H: Interstitial chemotherapy for malignant gliomas: the Johns Hopkins experience. J Neurooncol 2007, 83:61-70.

3. Parsons DW, Jones S, Zhang X, Lin JC, Leary RJ, Angenendt P, Mankoo P, Carter H, Siu IM, Gallia GL, Olivi A, McLendon R, Rasheed BA, Keir S,
Nikolskaya T, Nikolsky Y, Busam DA, Tekleab H, Diaz LA, Hartigan J, Smith DR, Strausberg RL, Marie SK, Shinjo SM, Yan H, Riggins GJ, Bigner DD, Karchin R, Papadopoulos N, Parmigiani G, Vogelstein B, Velculescu VE, Kinzler KW: An Integrated Genomic Analysis of Human Glioblastoma Multiforme. Science 2008, 321:1807-1812.

4. Prevarskaya N, Skryma R, Bidaux G, Flourakis M, Shuba Y: Ion channels in death and differentiation of prostate cancer cells. Cell Death Differ 2007, 14:1295-1304.

5. MacFarlane $\mathrm{SN}$, Sontheimer $\mathrm{H}$ : Changes in ion channel expression accompany cell cycle progression of spinal cord astrocytes. Glia 2000, 30:39-48.

6. Knutson P, Ghiani CA, Zhou JM, Gallo V, McBain CJ: K+ channel expression and cell proliferation are regulated by intracellular sodium and membrane depolarization in oligodendrocyte progenitor cells. J Neurosci 1997, 17:2669-2682.

7. Fiske JL, Fomin VP, Brown ML, Duncan RL, Sikes RA: Voltage-sensitive ion channels and cancer. Cancer Metastasis Rev 2006, 25:493-500.

8. Kunzelmann K: Ion channels and cancer. J Membr Biol 2005, 205:159-173.

9. Schonherr R: Clinical relevance of ion channels for diagnosis and therapy of cancer. J Membr Biol 2005, 205:175-184.

10. Fraser SP, Diss JK, Chioni AM, Mycielska ME, Pan H, Yamaci RF, Pani F, Siwy Z, Krasowska M, Grzywna Z, Brackenbury WJ, Theodorou D, Koyuturk M, Kaya H, Battaloglu E, De Bella MT, Slade MJ, Tolhurst R, Palmieri C, Jiang J, Latchman DS, Coombes RC, Djamgoz MB: Voltagegated sodium channel expression and potentiation of human breast cancer metastasis. Clin Cancer Res 2005, 11:5381-5389.

11. Smith JS, Tachibana I, Passe SM, Huntley BK, Borell TJ, Iturria N, O'Fallon JR, Schaefer PL, Scheithauer BW, James CD, Buckner JC, Jenkins RB: PTEN mutation, EGFR amplification, and outcome in patients with anaplastic astrocytoma and glioblastoma multiforme. J Natl Cancer Inst 2001, 93:1246-1256.

12. Goldin AG, Safa AR: Digitalis and cancer. Lancet 1984, 1:1134.

13. Mijatovic T, Van Quaquebeke E, Delest B, Debeir O, Darro F, Kiss R: Cardiotonic steroids on the road to anti-cancer therapy. Biochim Biophys Acta 2007, 1776:32-57.

14. Stenkvist B: Is digitalis a therapy for breast carcinoma? Oncol Rep 1999, 6:493-496

15. Wang JK, Portbury S, Thomas MB, Barney S, Ricca DJ, Morris DL, Warner DS, Lo DC: Cardiac glycosides provide neuroprotection against ischemic stroke: discovery by a brain slice-based compound screening platform. Proc Natl Acad Sci USA 2006, 103:10461-10466.

16. Yan H, Parsons DW, Jin G, McLendon R, Rasheed BA, Yuan W, Kos I, BatinicHaberle I, Jones S, Riggins GJ, Friedman H, Friedman A, Reardon D, Herndon J, Kinzler KW, Velculescu VE, Vogelstein B, Bigner DD: IDH1 and IDH2 mutations in gliomas. N Engl J Med 2009, 360:765-773.

17. Diss JK, Stewart D, Pani F, Foster CS, Walker MM, Patel A, Djamgoz MB: A potential novel marker for human prostate cancer: voltage-gated sodium channel expression in vivo. Prostate Cancer Prostatic Dis 2005, 8:266-273

18. Smith P, Rhodes NP, Shortland AP, Fraser SP, Djamgoz MB, Ke Y, Foster CS: Sodium channel protein expression enhances the invasiveness of rat and human prostate cancer cells. FEBS Lett 1998, 423:19-24.

19. Uysal-Onganer P, Djamgoz MB: Epidermal growth factor potentiates in vitro metastatic behaviour of human prostate cancer PC-3 M cells: involvement of voltage-gated sodium channel. Mol Cancer 2007, 6:76.

20. Lefranc F, Mijatovic T, Kondo Y, Sauvage S, Roland I, Debeir O, Krstic D, Vasic V, Gailly P, Kondo S, Blanco G, Kiss R: Targeting the alpha 1 subunit of the sodium pump to combat glioblastoma cells. Neurosurgery 2008, 62:211-221, discussion 221-212

21. Mijatovic T, Roland I, Van Quaquebeke E, Nilsson B, Mathieu A, Van Vynckt F, Darro F, Blanco G, Facchini V, Kiss R: The alpha1 subunit of the sodium pump could represent a novel target to combat non-small cell lung cancers. J Pathol 2007, 212:170-179.

22. Anderson JD, Hansen TP, Lenkowski PW, Walls AM, Choudhury IM, Schenck HA Friehling M, Holl GM, Patel MK, Sikes RA, Brown ML: Voltage-gated sodium channel blockers as cytostatic inhibitors of the androgen-independent prostate cancer cell line PC-3. Mol Cancer Ther 2003, 2:1149-1154.

doi:10.1186/1476-4598-10-17

Cite this article as: Joshi et al: Sodium ion channel mutations in glioblastoma patients correlate with shorter survival. Molecular Cancer 2011 10:17 